\title{
Nonparaxial singular beams inside the focal region of a high numerical-aperture lens
}

\author{
Vladlen G. Shvedov \\ Department of Physics, Taurida National V. Vernandsky University, 95007 \\ Simferopol, Crimea, Ukraine, vgs124@physics.anu.edu.au
}

Received: 18.03 .2011

\begin{abstract}
Key optical technologies, including lithography, data storage, optical tweezers, microscopy, and ultrafast laser materials processing, rely on strongly focused light beams. Such beams are often used to exploit a vectorial nature of light and so detailed knowledge of the field structure inside a tight focus becomes increasingly important. So far, theoretical studies of intra-focal optical field components have been mainly concentrated on spatially homogeneous states of light polarisation. In this work we present a new development in the calculations of local polarisation structure of tightly focused singular beams, including radially and azimuthally polarised hollow beams.
\end{abstract}

Keywords: singular beam, vector optical field, tight focus

PACS: 42.25.Ja, 42.60.Jf, 78.20.Bh

UDC: $535.51,535.316$

A rapidly increasing number of various practical applications in the last few years have attracted significant interest to studies related to three-dimensional structure of the field inside a focus of high numerical-aperture optical systems [1-5]. Experimental intrafocal mapping of the squared electric field components is usually indirectly achieved with near field probes [6], point scatterers [7], fluorescent molecules and beads [8, 9], and the knife edge method [10]. Recently, a new approach has been developed [2] for visualising nanoscale structure of the electric field inside the focal volume of tightly focused singular beams, which is based on permanent imprinting of the field in transparent media.

From the theoretical point of view, the first comprehensive studies of tightly focused linearly and circularly polarised Laguerre-Gaussian beams have been performed in Refs. [11-13]. In Refs. $[1,14]$, the authors have attempted to analyse the focal spot of both radially and azimuthally polarised beams. The problem has been qualitatively solved in Ref. [14] for the case of non-apodised cylindrical vector beams, hence providing basic understanding of the focal structure of real beams. The general method allowing one to solve such problems is based on Debye vector integrals. A complexity of the problem for some types of vector beams, e.g., radially or azimuthally polarised beams, is related to finding an integrand $\Psi$ which would satisfy the conditions for the paraxial waves $\left(\left(\nabla_{\perp}^{2}+4 \pi i \lambda^{-1} \partial_{z}\right) \Psi=0\right)$ and simultaneously describe the vector field components. In the above expression, $\nabla_{\perp}=\partial_{x} \mathbf{e}_{x}+\partial_{y} \mathbf{e}_{y}$ and $\partial_{u} \equiv \partial / \partial u$, with $\mathbf{e}_{x}$, $\mathbf{e}_{y}$ being the Cartesian unit vectors and $\lambda$ the wavelength of light.

In this work we wish to show that the complete vector function for radially or azimuthally polarised beams can be obtained by first finding solutions for linearly and circularly polarised optical vortices, in which case the choice of the integrand $\Psi$. does not pose any difficulties. We start 
with finding the solutions of the Debye problem for linearly polarised optical vortices, following the formalism suggested in Refs. [11,12].

The complex amplitude of a scalar Laguerre-Gaussian beam at its waist may be written as [12]

$$
E_{0}(r, \varphi, z=0)=A_{0}\left(\sqrt{2} r / w_{0}\right)^{|m|} L_{p}^{|m|}\left(2 r^{2} / w_{0}^{2}\right) \exp \left(-r^{2} / w_{0}^{2}\right) \exp (\operatorname{im} \varphi),
$$

where $A_{0}$ is the characteristic amplitude at the peak of intensity, $r$ the radial distance from the beam axis, $\varphi$ the azimuthal angle in the entrance plane (see Fig. 1), $L_{p}^{|m|}(x)$ the Laguerre polynomial with the radial index $p$ and the azimuthal index $m$, and $w_{0}$ the beam waist.

The radial coordinate can also be presented as $r=f \sin (\theta)$, where $\theta$ is the angle between the optical axis and the light ray (see Fig. 1). Then the ratio $r / w_{0}$ becomes

$$
\frac{r}{w}=\frac{a}{w} \frac{1}{N A} \sin \theta=\gamma \frac{\sin \theta}{\sin \alpha} .
$$

Here $N A=a / f=\sin \alpha$ stands for the numerical aperture of the focusing lens (from now on, the refractive index of the medium in which the beam is focused is assumed to be equal to unity), $\alpha$ is the maximum angle defining the beam convergence $\left(\alpha=\theta_{\max }\right)$, and the ratio $\gamma=a / w$ of the entrance aperture radius $a$ and the beam waist $w$. represents the truncation parameter denoting the function of the beam inside the physical aperture. It defines the fraction of the beam which goes through the lens. The lens is overfilled if $\gamma<1$ and underfilled if $\gamma>1[11,12]$.

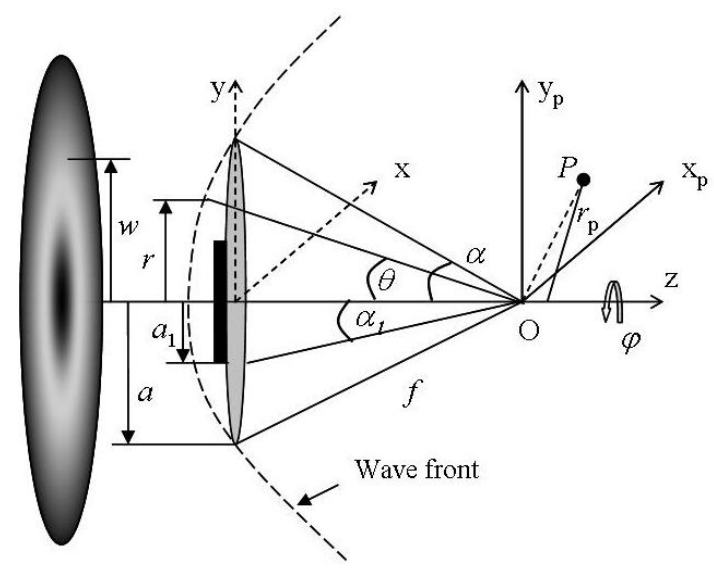

Fig. 1. Representation of focal geometry for singular beams. $(x, y)$ and $\left(x_{p}, y_{p}\right)$ are the Cartesian coordinates in a plane of physical aperture and a focal plane, respectively, $r_{p}$ denotes the radial distance of point $P$ from $z$ axis, $a$ the physical aperture, $a_{1}$ the radius of non-transparent disk which increases efficiency of the physical aperture, $\alpha$ the numerical aperture (NA) of an objective, $\alpha_{1}$ the angular dimension of circular beam stop of radius $a_{1}, w$ the parameter defining size of an incident beam, and $f$ the focal length.

In our case, which deals with an optical vortex incident on a lens, the radial index $p=0$. Upon taking Eq. (2) into account, Eq. (1) may be written as [14]

$$
E_{0}(r, \varphi, z=0)=A_{1}(\theta) \exp (i m \varphi),
$$

where

$$
A_{1}(\theta)=A_{0}\left(\sqrt{2} \gamma \frac{\sin \theta}{\sin \alpha}\right)^{|m|} \exp \left(-\gamma^{2} \frac{\sin ^{2} \theta}{\sin ^{2} \alpha}\right)
$$


describes the amplitude function of a collimated optical vortex. According to the focusing geometry shown in Fig. 1, the field distribution in the focal region is given by

$$
\begin{aligned}
E\left(r_{P}, z\right) & =(-i A / \lambda) \int_{\alpha_{1}}^{\alpha} \int_{0}^{2 \pi} A_{1}(\theta) P(\theta, \varphi) A_{2}(\theta) \exp (i m \varphi) \\
& \times \exp \left(-i k r_{P} \sin \theta \cos \left(\varphi-\varphi_{P}\right)\right) \exp (-i k z \cos \theta) \sin \theta d \theta d \varphi,
\end{aligned}
$$

where $A_{1}$ is a coefficient that depends on the optical parameters of the system [6], $\lambda$ the light wavelength, the term $P(\theta, \varphi)$ defines the polarisation distribution [4] at the entrance pupil, and $A_{2}$ gives the apodising function which is equal to $\sqrt{\cos \theta}$ for aplanatic lenses [15].

The polarisation distribution of the incident beam in the entrance pupil plane may be presented as $[4,12]$

$$
\begin{aligned}
P(\theta, \varphi)= & b_{1}(\cos \theta \cos \varphi \sin \varphi-\sin \varphi \cos \varphi) \\
& +b_{2}\left(\cos \theta \sin ^{2} \varphi+\cos ^{2} \varphi\right)-b_{1} \sin \theta \cos \varphi-b_{2} \sin \theta \sin \varphi,
\end{aligned}
$$

where $b_{1}$ and $b_{2}$ denote the 'strengths' of the $x$ - and $y$-linearly polarised incident beams, respectively. Then Eq. (4) can be reduced to a single integral by using the identity

$$
\int_{0}^{2 \pi} \exp (i m \varphi) \exp \left(-i k r_{P} \sin \theta \cos \left(\varphi-\varphi_{P}\right)\right) d \varphi=2 \pi i^{m} J_{m}\left(k r_{P} \sin \theta\right) \exp \left(i m \varphi_{P}\right),
$$

where $J_{m}\left(k r_{P} \sin \theta\right)$ is the Bessel function of the first kind and the $m$ th order.

Taking into consideration the above arguments, one can write the electric field components in the focal region of the $x$-polarised vortex beam as [12]

$$
\begin{aligned}
& E_{x}=2 \pi i^{m} \exp \left(i m \varphi_{P}\right) I_{m}-\pi i^{m+2} \exp \left(i(m+2) \varphi_{P}\right) I_{m+2}-\pi i^{m-2} \exp \left(i(m-2) \varphi_{P}\right) I_{m-2}, \\
& E_{y}=-i \pi i^{m-2} \exp \left(i(m-2) \varphi_{P}\right) I_{m-2}+i \pi i^{m+2} \exp \left(i(m+2) \varphi_{P}\right) I_{m+2}, \\
& E_{z}=-2 \pi i^{m+1} \exp \left(i(m+1) \varphi_{P}\right) I_{m+1}-2 \pi i^{m-1} \exp \left(i(m-1) \varphi_{P}\right) I_{m-1} .
\end{aligned}
$$

For comparison, when the incident vortex is $y$-polarised, the components of its electric field in the focal region are given by

$$
\begin{aligned}
& E_{x}=-i \pi i^{m-2} \exp \left(i(m-2) \varphi_{P}\right) I_{m-2}+i \pi i^{m+2} \exp \left(i(m+2) \varphi_{P}\right) I_{m+2}, \\
& E_{y}=2 \pi i^{m} \exp \left(i m \varphi_{P}\right) I_{m}+\pi i^{m+2} \exp \left(i(m+2) \varphi_{P}\right) I_{m+2}+\pi i^{m-2} \exp \left(i(m-2) \varphi_{P}\right) I_{m-2}, \\
& E_{z}=-i 2 \pi i^{m-1} \exp \left(i(m-1) \varphi_{P}\right) I_{m-1}+i 2 \pi i^{m+1} \exp \left(i(m+1) \varphi_{P}\right) I_{m+1} .
\end{aligned}
$$

Here

$$
\begin{aligned}
& I_{m}\left(r_{P}, z\right)=(-i A / 2 \lambda) \int_{\alpha_{1}}^{\alpha} A_{1}(\theta) \sqrt{\cos \theta}(1+\cos \theta) J_{m}\left(k r_{P} \sin \theta\right) \exp (-i k z \cos \theta) \sin \theta d \theta, \\
& I_{m \pm 1}\left(r_{P}, z\right)=(-i A / 2 \lambda) \int_{\alpha_{1}}^{\alpha} A_{1}(\theta) \sqrt{\cos \theta} J_{m \pm 1}\left(k r_{P} \sin \theta\right) \exp (-i k z \cos \theta) \sin ^{2} \theta d \theta, \\
& I_{m \pm 2}\left(r_{P}, z\right)=(-i A / 2 \lambda) \int_{\alpha_{1}}^{\alpha} A_{1}(\theta) \sqrt{\cos \theta}(1-\cos \theta) J_{m \pm 2}\left(k r_{P} \sin \theta\right) \exp (-i k z \cos \theta) \sin \theta d \theta .
\end{aligned}
$$

The analysis of the above expressions shows that all of the three electric field components are present in the focal region of the optical vortex and the light intensity is generally nonzero at the optical axis: 


$$
I=\left|E_{x}\left(r_{P}, z\right)\right|^{2}+\left|E_{y}\left(r_{P}, z\right)\right|^{2}+\left|E_{z}\left(r_{P}, z\right)\right|^{2} .
$$

The solutions found above also allow one to examine focusing of elliptically polarised optical vortices with the complex field amplitude in the entrance pupil plane given by:

$$
\mathbf{E}(\theta, \varphi)=A_{1}(\theta) \exp (\operatorname{im} \varphi)\left(\mathbf{E}_{x}+e^{i \phi} \mathbf{E}_{y}\right),
$$

where $\mathbf{E}_{x}$ and $\mathbf{E}_{y}$ stand for the $x$ and $y$ vector field components and $\phi$ is the phase shift between them. (Notice that the bold type indicates a vector variable). A circularly polarised vortex is produced when $\left|E_{x}\right|=\left|E_{y}\right|$ and $\phi= \pm \pi / 2$.

The following sign convention for the circularly polarised beams is adopted hereafter: the circular polarisation is referred to as right-handed if $\phi=+\pi / 2$ and left-handed if $\phi=-\pi / 2$, i.e. $\mathbf{E}^{ \pm}=\mathbf{E}_{x} \pm i \mathbf{E}_{y}=\sqrt{2} E \boldsymbol{\sigma}^{ \pm}$. Here $\boldsymbol{\sigma}^{ \pm}=(\overline{\mathbf{x}} \pm i \quad \overline{\mathbf{y}}) / \sqrt{2}$ denotes the unit vector of circular polarisation and $\overline{\mathbf{x}}, \overline{\mathbf{y}}$ the unit Cartesian vectors.

Using the sign convention introduced we can add the field components in Eqs. (7) and (8) taking into account the phase shift given by Eq. (9). Then the Cartesian components of the righthanded field in the vicinity of the focus take the following forms:

$$
\begin{gathered}
E_{x}^{+}=2 \pi i^{m} \exp \left(i m \varphi_{P}\right) I_{m}-2 \pi i^{m+2} \exp \left(i(m+2) \varphi_{P}\right) I_{m+2}, \\
E_{y}^{+}=i 2 \pi i^{m} \exp \left(i m \varphi_{P}\right) I_{m}+i 2 \pi i^{m+2} \exp \left(i(m+2) \varphi_{P}\right) I_{m+2}, \\
E_{z}^{+}=-4 \pi i^{m+1} \exp \left(i(m+1) \varphi_{P}\right) I_{m+1} .
\end{gathered}
$$

Similarly, for the left-handed light field we obtain

$$
\begin{gathered}
E_{x}^{-}=2 \pi i^{m} \exp \left(i m \varphi_{P}\right) I_{m}-2 \pi i^{m-2} \exp \left(i(m-2) \varphi_{P}\right) I_{m-2}, \\
E_{y}^{-}=-i 2 \pi i^{m} \exp \left(i m \varphi_{P}\right) I_{m}-i 2 \pi i^{m-2} \exp \left(i(m-2) \varphi_{P}\right) I_{m-2}, \\
E_{z}^{-}=-4 \pi i^{m-1} \exp \left(i(m-1) \varphi_{P}\right) I_{m-1} .
\end{gathered}
$$

Using the same notations, we get

$$
\begin{aligned}
& E_{x}^{ \pm}=2 \pi i^{m} \exp \left(i m \varphi_{P}\right) I_{m}-2 \pi i^{m \pm 2} \exp \left(i(m \pm 2) \varphi_{P}\right) I_{m \pm 2}, \\
& E_{y}^{ \pm}= \pm 2 i\left(\pi i^{m} \exp \left(i m \varphi_{P}\right) I_{m}+\pi i^{m \pm 2} \exp \left(i(m \pm 2) \varphi_{P}\right) I_{m \pm 2}\right) \\
& E_{z}^{ \pm}=-4 \pi i^{m \pm 1} \exp \left(i(m \pm 1) \varphi_{P}\right) I_{m \pm 1}
\end{aligned}
$$

for the both vortices.

These relations allow one to deduce the salient features of the electric field in the focal region. For instance, the fundamental Gaussian beam at the focal point $\left(z=0, r_{P}=0\right)$ always retains the initial state of polarisation [11]. At the same time, the optical vortices with the topological charges $m=1,2$ have in general nonzero electric fields at this point, due to contribution from the longitudinal components of the field. When the topological charge is greater than three (i.e., $m \geq 3$ ), the focal intensity is always zero at the centre of the beam $[11,16]$. Of particular interest is the case of circularly polarised vortices with $m= \pm 1$. According to Eqs. (10), the vortex preserves zero intensity along the whole optical axis when the sign of the topological charge coincides with that of the handedness $(m=\sigma)$. When the charge and the polarisation have the opposite signs $(m=-\sigma)$, an intensity maximum appears at the focus, owing to the longitudinal component of the electric field $E_{z}$ (see Fig. 2a). A detailed analysis of the properties of circularly polarised, and more complex singular, modes is easier to perform using the polar basic vectors of the circular cylindrical coordinates (see Eq. (9)): 


$$
\begin{aligned}
\overline{\mathbf{e}}_{r} & =\overline{\mathbf{x}} \cos \varphi+\overline{\mathbf{y}} \sin \varphi, \\
\overline{\mathbf{e}}_{\varphi} & =\overline{\mathbf{y}} \cos \varphi-\overline{\mathbf{x}} \sin \varphi,
\end{aligned}
$$

where $\overline{\mathbf{e}}_{r}$ and $\overline{\mathbf{e}}_{\varphi}$ are the unit vectors along the radial and azimuthal directions.

The field components in Eq. (9) transform to the new coordinate system as follows:

$$
\begin{aligned}
& E_{r}^{ \pm}=2 \pi i^{m} \exp \left(i(m \pm 1) \varphi_{P}\right) I_{m}-2 \pi i^{m \pm 2} \exp \left(i(m \pm 1) \varphi_{P}\right) I_{m \pm 2}, \\
& E_{\varphi}^{ \pm}= \pm 2 i\left(\pi i^{m} \exp \left(i(m \pm 1) \varphi_{P}\right) I_{m}+\pi i^{m \pm 2} \exp \left(i(m \pm 1) \varphi_{P}\right) I_{m \pm 2}\right), \\
& E_{z}^{ \pm}=-4 \pi i^{m \pm 1} \exp \left(i(m \pm 1) \varphi_{P}\right) I_{m \pm 1} .
\end{aligned}
$$

After simplification they can be rewritten as

$$
\begin{aligned}
& E_{r}^{ \pm}=2 \pi i^{m}\left(I_{m}+I_{m \pm 2}\right) \exp \left(i(m \pm 1) \varphi_{P}\right), \\
& E_{\varphi}^{ \pm}= \pm 2 i \pi i^{m}\left(I_{m}-I_{m \pm 2}\right) \exp \left(i(m \pm 1) \varphi_{P}\right), \\
& E_{z}^{ \pm}=-4 \pi i^{m \pm 1} \exp \left(i(m \pm 1) \varphi_{P}\right) I_{m \pm 1} .
\end{aligned}
$$

In order to obtain solutions for the radial and azimuthal modes at the focus, we use the following reasoning. Radial and azimuthal beams with coinciding waists and the same divergences acquired while propagating along the optical axis $z$ of a focusing system, can be presented as

$$
\mathbf{E}_{T M}=\left(\sqrt{2} r / w_{0}\right) G \overline{\mathbf{e}}_{r}, \mathbf{E}_{T E}=\left(\sqrt{2} r / w_{0}\right) G \overline{\mathbf{e}}_{\varphi},
$$

where $G=A_{0} \exp \left(-r^{2} / w_{0}^{2}\right)$ describes the fundamental Gaussian beam given by Eq. (1).

The circularly polarised optical vortices with a zero orbital angular momentum ( $\sigma= \pm 1 ; m=\mp 1)$ may be presented as a superposition of the two modes given by Eqs. (13) [17, 18]:

$$
\mathbf{E}_{m=\mp 1}^{\sigma= \pm 1}=\mathbf{E}_{T M} \pm i \mathbf{E}_{T E}=\left(2 r / w_{0}\right) G \boldsymbol{\sigma}^{ \pm} \exp (\mp i \varphi),
$$

where $\boldsymbol{\sigma}^{ \pm}=\frac{1}{\sqrt{2}}\left(\overline{\mathbf{e}}_{r} \pm i \quad \overline{\mathbf{e}}_{\varphi}\right) \exp ( \pm i \varphi)$ is the unit vector of circular polarisation in the cylindrical coordinates. Hence, by knowing the focal field distribution of the circularly polarised vortices (12) it is possible to reconstruct, with the aid of Eq. (14), the field of the corresponding radial and azimuthal modes. Indeed, from the system of equations

we find

$$
\left\{\begin{array}{l}
\mathbf{E}_{T M}+i \mathbf{E}_{T E}=\mathbf{E}_{m=-1}^{\sigma=+1} \\
\mathbf{E}_{T M}-i \mathbf{E}_{T E}=\mathbf{E}_{m=+1}^{\sigma=-1}
\end{array}\right.
$$

$$
\left\{\begin{array}{l}
\mathbf{E}_{T M}=\frac{1}{2}\left(\mathbf{E}_{m=-1}^{\sigma=+1}+\mathbf{E}_{m=+1}^{\sigma=-1}\right) \\
\mathbf{E}_{T E}=\frac{1}{2 i}\left(\mathbf{E}_{m=-1}^{\sigma=+1}-\mathbf{E}_{m=+1}^{\sigma=-1}\right)
\end{array} .\right.
$$

In this case the fields given by Eqs. (12) may be expressed as

$$
\begin{array}{ll}
E_{r, m=-1}^{\sigma=+1}=-2 \pi i\left(I_{m=-1}+I_{1}\right), & E_{r, m=+1}^{\sigma=-1}=2 \pi i\left(I_{m=+1}+I_{-1}\right), \\
E_{\varphi, m=-1}^{\sigma=+1}=-2 \pi i\left(I_{m=-1}-I_{1}\right), & E_{\varphi, m=+1}^{\sigma=-1}=-2 \pi i\left(I_{m=+1}-I_{-1}\right), \\
E_{z, m=-1}^{\sigma=+1}=-4 \pi I_{0}, & E_{z, m=+1}^{\sigma=-1}=-4 \pi I_{0}
\end{array}
$$

where 


$$
\begin{aligned}
& I_{m=-1}\left(r_{P}, z\right)=(-i A / 2 \lambda) \int_{\alpha_{1}}^{\alpha} A_{1}(\theta) \sqrt{\cos \theta}(1+\cos \theta) J_{-1}\left(k r_{P} \sin \theta\right) \exp (-i k z \cos \theta) \sin \theta d \theta \\
& I_{m=+1}\left(r_{P}, z\right)=(-i A / 2 \lambda) \int_{\alpha_{1}}^{\alpha} A_{1}(\theta) \sqrt{\cos \theta}(1+\cos \theta) J_{1}\left(k r_{P} \sin \theta\right) \exp (-i k z \cos \theta) \sin \theta d \theta \\
& I_{0}\left(r_{P}, z\right)=(-i A / 2 \lambda) \int_{\alpha_{1}}^{\alpha} A_{1}(\theta) \sqrt{\cos \theta} J_{0}\left(k r_{P} \sin \theta\right) \exp (-i k z \cos \theta) \sin ^{2} \theta d \theta \\
& I_{1}\left(r_{P}, z\right)=(-i A / 2 \lambda) \int_{\alpha_{1}}^{\alpha} A_{1}(\theta) \sqrt{\cos \theta}(1-\cos \theta) J_{1}\left(k r_{P} \sin \theta\right) \exp (-i k z \cos \theta) \sin \theta d \theta \\
& I_{-1}\left(r_{P}, z\right)=(-i A / 2 \lambda) \int_{\alpha_{1}}^{\alpha} A_{1}(\theta) \sqrt{\cos \theta}(1-\cos \theta) J_{-1}\left(k r_{P} \sin \theta\right) \exp (-i k z \cos \theta) \sin \theta d \theta
\end{aligned}
$$

By applying the well-known identity $J_{-1}(x)=-J_{1}(x)$ to the above integrals for the circularly polarised single-charged vortices, one can reduce Eqs. (16) to the following:

$$
\begin{array}{ll}
E_{r, m=-1}^{\sigma=+1}=2 \pi i I_{r}, & E_{r, m=+1}^{\sigma=-1}=2 \pi i I_{r}, \\
E_{\varphi, m=-1}^{\sigma=+1}=4 \pi i I_{\varphi}, & E_{\varphi, m=+1}^{\sigma=-1}=-4 \pi i I_{\varphi}, \\
E_{z, m=-1}^{\sigma=+1}=-4 \pi I_{0}, & E_{z, m=+1}^{\sigma=-1}=-4 \pi I,
\end{array}
$$

where

$$
\begin{aligned}
& I_{r}=(-i A / 2 \lambda) \int_{\alpha_{1}}^{\alpha} A_{1}(\theta) \sqrt{\cos \theta} J_{1}\left(k r_{P} \sin \theta\right) \exp (-i k z \cos \theta) \sin 2 \theta d \theta, \\
& I_{\varphi}=(-i A / 2 \lambda) \int_{\alpha_{1}}^{\alpha} A_{1}(\theta) \sqrt{\cos \theta} J_{1}\left(k r_{P} \sin \theta\right) \exp (-i k z \cos \theta) \sin \theta d \theta, \\
& I_{0}=(-i A / 2 \lambda) \int_{\alpha_{1}}^{\alpha} A_{1}(\theta) \sqrt{\cos \theta} J_{0}\left(k r_{P} \sin \theta\right) \exp (-i k z \cos \theta) \sin ^{2} \theta d \theta .
\end{aligned}
$$

Substituting Eqs. (17) into Eqs. (15), we find the electric field components of the focused radial and azimuthal modes:

$$
E_{r(T M)}=4 \pi i I_{r}, E_{\varphi(T M)}=0, E_{z(T M)}=-8 \pi I_{0}, E_{r(T E)}=0, E_{\varphi(T E)}=8 \pi i I_{\varphi}, E_{z(T E)}=0 .
$$

In the vector representation, the focal field components of the radial and azimuthal modes become as follows:

$$
\left\{\begin{array}{l}
\mathbf{E}_{T M}=4 \pi\left(i I_{r} \overline{\mathbf{e}}_{r}-2 I_{0} \overline{\mathbf{e}}_{z}\right) \\
\mathbf{E}_{T E}=8 \pi i I_{\varphi} \overline{\mathbf{e}}_{\varphi}
\end{array} .\right.
$$

One can easily see from these expressions that the azimuthal mode preserves both its polarisation and zero intensity along the whole optical axis even in the regime of tight focusing, whereas the intensity and the polarisation structure of the radial mode undergo significant transformations (see Fig. 2).

This difference in the behaviours of the two modes inside the tight focus can be exploited when solving various practical problems. For instance, conservation of the zero intensity in the azimuthal mode facilitates significantly optical implosion of materials. This novel phenomenon has recently been demonstrated in the experiments on compressing fused silica with double-charge femtosecond vortex laser pulses $[19,20]$. On the other hand, the longitudinal component of the electric field is also crucial for some important applications [5]. Radially polarised beams focused with high numerical-aperture optics are now routinely used in microscopy [21], second-harmonic generation [22, 23], Raman spectroscopy [24], and particle trapping or manipulation [25]. 

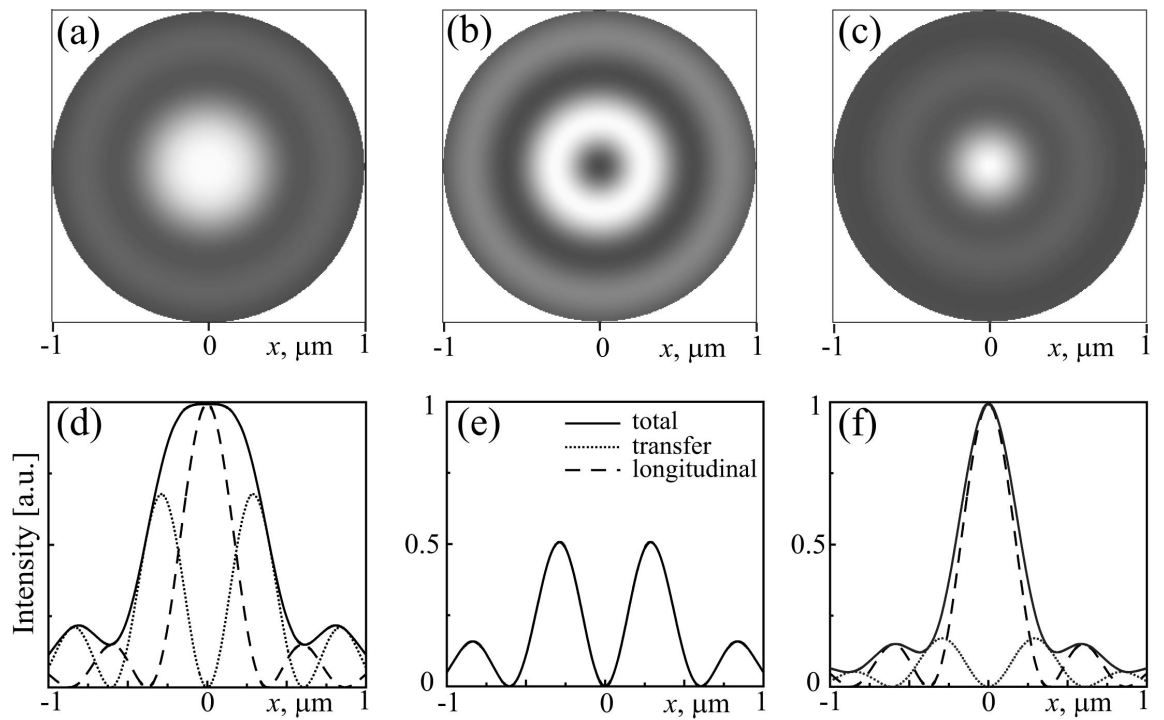

Fig. 2. Simulated intensity distributions in the focal plane for an optical vortex $m= \pm 1 ; \sigma=\mp 1$ ((a) and (d)) and azimuthally ((b) and (e)) or radially ((c) and (f)) polarised beams. Solid lines in (d)-(f) show total intensity distribution, dotted lines correspond to intensity distributions in the transferred polarisation state, and dashed lines give intensity distributions for the longitudinal polarisation state inside the beams. The parameters of the optical system and the beams are as follows: $\mathrm{NA}=0.9, \lambda=780 \mathrm{~nm}, a_{1}=1.1 \mathrm{~mm}$, and $a=2 \mathrm{~mm}$.

\section{Acknowledgement}

I thank Dr. Cyril Hnatovsky and Prof. Alexander Volyar for useful discussions.

\section{References}

1. Wang H, Shi L, Lukyanchuk B, Sheppard C and Chong C, 2008. Creation of a needle of longitudinally polarized light in vacuum using binary optics. Nature Photonics. 2: 501-505.

2. Hnatovsky C, Shvedov V, Krolikowski W and Rode A, 2011. Revealing local field structure of focused ultrashort pulses. Phys. Rev. Lett. 106: 123901-05.

3. Wang Z, Guo W, Li L, Luk'yanchuk B, Khan A, Liu Z, Chen Z and Hong Z, 2011. Optical virtual imaging at $50 \mathrm{~nm}$ lateral resolution with a white-light nanoscope. Nature Commun. 2: 218-222.

4. Helseth L, 2006. Smallest focal hole, Opt. Comm. 257: 1-8.

5. Zhan Q, 2009. Cylindrical vector beams: from mathematical concepts to applications. Adv. Opt. Photonics. 1: 1-57.

6. Rhodes S, Nugent K and Roberts A, 2002. Precision measurement of the electromagnetic fields in the focal region of a high-numerical-aperture lens using a tapered fiber probe. J. Opt. Soc. Amer. A. 19: 1689-1693.

7. Wilson T, Juškaitis R and Higdon P, 1997. The imaging of dielectric point scatterers in conventional and confocal polarisation microscopes. Opt. Commun. 141: 298-313.

8. Novotny L, Beversluis M, Youngworth K and Brown T, 2001. Longitudinal field modes probed by single molecules. Phys. Rev. Lett. 86: 5251-5254.

9. Bokor N, Iketaki Y, Watanabe T and Fujii M, 2005. Investigation of polarization effects for high-numerical-aperture first-order Laguerre-Gaussian beams by 2D scanning with a single fluorescent microbead. Opt. Express. 13: 10440-10447. 
10. Dorn R, Quabis S and Leuchs G, 2003. Sharper focus for a radially polarized light beam. Phys. Rev. Lett. 91: 233901-233905.

11. Torok P and Munro P, 2004. The use of Gauss-Laguerre vector beams in STED microscopy. Opt. Express. 12: 3605-3617.

12. Singh R, Senthilkumaran $P$ and Singh K, 2008. Effect of primary spherical aberration on highnumerical aperture of a Laguerre-Gaussian beam. J. Opt. Soc. Amer. A. 25: 1307-1318.

13. Volyar A, Shvedov V and Fadeyeva T, 2001. The structure of a nonparaxial Gaussian beam near the focus: II. Optical vortices. Opt. Spectrosc. 90 (1): 93-100.

14. Youngworth $\mathrm{K}$ and Brown T, 2000. Focusing of high numerical aperture cylindrical vector beams. Opt. Express. 7: 77-87.

15. Richards B and Wolf E, 1959. Electromagnetic diffraction in the optical systems. 2. Structure of the image field in an aplanatic system. Proc. Roy. Soc. London, Ser. A. 253: 358-379.

16. Izdebskaya Y, Shvedov V and Volyar A, 2005. Focusing of wedge-generated higher-order optical vortices. Opt. Lett. 30: 2530-2532.

17. Fadeyeva T, Shvedov V, Izdebskaya Y, Volyar A, Brasselet E, Neshev D, Desyatnikov A, Krolikowski W and Kivshar Y, 2010. Spatially engineered polarization states and optical vortices in uniaxial crystals. Opt. Express. 30: 10848-10863.

18. Fadeyeva T, Shvedov V, Shostka N, Alexeyev C and Volyar A, 2010. Natural shaping of the cylindrically polarized beams. Opt. Lett. 35: 3787-3789.

19. Shvedov V, Hnatovsky C, Krolikowski W and Rode A, 2010. Efficient beam converter for the generation of high-power femtosecond vortices. Opt. Lett. 35: 2660-2662.

20. Hnatovsky C, Shvedov V, Krolikowski W and Rode A, 2010. Materials processing with a tightly focused femtosecond laser vortex pulse. Opt. Lett. 35: 3417-3419.

21. Watanabe K, Horiguchi N and Kano H, 2007. Optimized measurement probe of the localized surface plasmon microscope by using radially polarized illumination. Appl. Opt. 46: 49854990.

22. Biss D and Brown T, 2003. Polarization-vortex-driven second-harmonic generation. Opt. Lett. 28: 923-925.

23. Yew E and Sheppard C, 2007. Second harmonic generation polarization microscopy with tightly focused linearly and radially polarized beams. Opt. Commun. 275: 453-457.

24. Hayazawa N, Saito Y and Kawata S, 2004. Detection and characterization of longitudinal field for tip-enhanced Raman spectroscopy. Appl. Phys. Lett. 85: 6239-6341.

25. Zhan Q, 2004. Trapping metallic Rayleigh particles with radial polarization. Opt. Express. 12: 3377-3382.

V.G. Shvedov, 2011. Nonparaxial singular beams inside the focal region of a high numericalaperture lens Ukr.J.Phys.Opt. 12: 109-116.

Анотація Основні оптичні технології, зокрема такі як літографія, запис даних, використання оптичних пінцетів, мікроскопія та надшвидка обробка лазерних матеріалів, базуються на сильному фокусуванні світлових променів. Використання ичих променів здебільшого базується на векторній природі світла, тому детальна інформація про структуру поля всередині фокуса є особливо важливою. Однак теоретичне дослідження внутрішньо фокусних оптичних компонент поля досі переважно конщентрувалося на просторово однорідних станах поляризачї̈ світла. У даній роботі представлено нові результати розрахунку структури локальної поляризачії сильно сфокусованого сингулярного променя, включаючи випадки радіально та азимутально поляризованого пустотілого променя. 